\title{
Genome resequencing data for Iranian local dogs and wolves
}

\author{
Zeinab Amiri Ghanatsaman 1,2, Guo-Dong Wang ${ }^{3}$, Masood Asadi Fozi ${ }^{1}$, Ya-Ping Zhang ${ }^{4}$ \\ and Ali Esmailizadeh ${ }^{1,3^{*}}$ (1)
}

\begin{abstract}
Objective: The data provided herein represent the whole-genome resequencing data related to three wolves and three Iranian local dogs. The understanding of genome evolution during animal domestication is an interesting subject in genome biology. Dog is an excellent model for understanding of domestication due to its considerable variety of behavioral and physical traits. The Zagros area of current day Iran has been identified as one of the initial centers of animal domestication. The availability of the complete genome sequences of Iranian local canids can be a valuable resource for researchers to address questions and testing hypotheses on the dog domestication process.

Data description: We collected blood samples from six Iranian local canids including two hunting dogs (Saluki breed), a mastiff dog (Qahderijani ecotype) and three wolves. We extracted genomic DNA from blood samples. Sequence data were produced using the Illumina HiSeq 2500 system. All sequence data are available in the National Genomics Data Center (NGDC), Genome Sequence Archive (GSA) database under the accession of CRA001324 and the National Center for Biotechnology Information (NCBI) under the accession of PRJNA639312. The short-read sequences with the mean depth of 16X were aligned to the dog reference genome (CanFam3.1) and achieved 99\% coverage of the reference assembly. The obtained information from this experiment will be useful in evolutionary biology.
\end{abstract}

Keywords: Whole-genome resequencing, Canid, Iran

\section{Objective}

Dogs (Canis familiaris) were probably the earliest domesticated animals and one of the human companions in ancient times [1, 2]. Archaeological findings and genetic research indicated that the dog breeds have derived from wild wolves [3-5]. In the Southwest Asia, major-scale farming extended within the so-named Fertile Crescent (FC), where the independent domestication of plants and animals occurred [6, 7]. Extensively, cultural advances occurred in the Zagros area of current day Iraq and Iran, connecting Iranian plateau and Mesopotamia

\footnotetext{
*Correspondence: aliesmaili@uk.ac.ir

1 Department of Animal Science, Faculty of Agriculture, Shahid Bahonar

University of Kerman, PB 76169-133 Kerman, Iran

Full list of author information is available at the end of the article
}

[8]. Dogs had been pictured frequently in Southwest Asia $[1,9]$. Consequently, one of the notable viewpoints on the primary location of the dog domestication has been the Southwest Asia, likely the Middle East [1]. Moreover, the Middle East has been included in the considerable allelic distribution between dog breeds and wolf [10]; however, this presumption has been queried because of dog-wolf hybridization as stated in previous studies [11-13]. The $\mathrm{dog}$ is a considerable example of phenotypic variation under artificial selection and demographic forces, but genetic basis of this diversity is not yet completely clear. Therefore, the availability of complete whole-genome resequencing data of Iranian local canids will provide an opportunity for researchers to trace the origin of dog domestication. We firstly carried out genome sequencing of six Iranian local canids including two hunting dogs

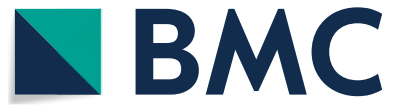

(c) The Author(s) 2020. This article is licensed under a Creative Commons Attribution 4.0 International License, which permits use, sharing, adaptation, distribution and reproduction in any medium or format, as long as you give appropriate credit to the original author(s) and the source, provide a link to the Creative Commons licence, and indicate if changes were made. The images or other third party material in this article are included in the article's Creative Commons licence, unless indicated otherwise in a credit line to the material. If material is not included in the article's Creative Commons licence and your intended use is not permitted by statutory regulation or exceeds the permitted use, you will need to obtain permission directly from the copyright holder. To view a copy of this licence, visit http://creativeco mmons.org/licenses/by/4.0/. The Creative Commons Public Domain Dedication waiver (http://creativecommons.org/publicdomain/ zero/1.0/) applies to the data made available in this article, unless otherwise stated in a credit line to the data. 
(Saluki breed), a mastiff dog (Qahderijani ecotype) and three wolves (Table 1). We used these data for identifying effective genomic variants in dogs and wolves [14].

\section{Data description}

We collected blood samples from three Iranian local dogs and three Iranian local wolves with the approval of the owners from six various sites in Iran. Sampling of Saluki dogs was done on Jamil Tavanaei's personal farms in Kurdistan zone (Sanandaj and Bijar) and sampling of a Qahderijani dog was conducted on Alireza Hoseini private farm in Isfahan zone. One of the wolf samples was collected from Kerman zoological garden in
Kerman zone and the others were collected from Eram zoological garden in Tehran zone. DNA was extracted with phenol/chloroform method. For sequencing library preparation, the genomic DNA was sheared to fragments of 300-500 bp, which were then end-repaired, "A"-tailed, and ligated to Illumina sequencing adapters. The ligated products with sizes of 400-500 bp were selected on $2 \%$ agarose gels and then amplified by LM-PCR. Illumina paired-end whole-genome resequencing for six individuals was done with Hiseq2500 Illumina system) http:// www.berrygenomics.com). Both nuclear and mitochondrial genomes were sequenced. We created $287.5 \mathrm{~Gb}$ data with a uniform read length of $150 \mathrm{bp}$. A total of

\section{Table 1 Overview of whole-genome sequence data files of six Iranian canids}

\begin{tabular}{|c|c|c|c|}
\hline Label & Name of data file/data set & File types(extension) & Data repository and identifier (DOI or accession number) \\
\hline Bioproject [24] & $\begin{array}{l}\text { Whole genome resequencing of the } \\
\text { Iranian native dogs and wolves }\end{array}$ & No file type & $\begin{array}{l}\text { PRJCA00118 } \\
\text { https://bigd.big.ac.cn/bioproject/browse/PRJCA001183 }\end{array}$ \\
\hline Data file 1 [25-28] & $\begin{array}{l}\text { YPi2985_L4_1_clean.fa.gz } \\
\text { YPi2985_L4_2_clean.fa.gz } \\
\text { YPi2985_L5_1_clean.fa.gz } \\
\text { YPi2985_L5_2_clean.fa.gz } \\
\text { YPi2985_L7_1_clean.fa.gz } \\
\text { YPi2985_L7_2_clean.fa.gz } \\
\text { YPi2985_L8_1_clean.fa.gz } \\
\text { YPi2985_L8_2_clean.fa.g }\end{array}$ & FASTQ (fq.gz) & $\begin{array}{l}\text { NGDC, Genome Sequence Archive https://bigd.big.ac.cn/ } \\
\text { gsa/browse/CRA001324/CRR042720 } \\
\text { NGDC, Genome Sequence Archive } \\
\text { https://bigd.big.ac.cn/gsa/browse/CRA001324/CRR042721 } \\
\text { NGDC, Genome Sequence Archive } \\
\text { https://bigd.big.ac.cn/gsa/browse/CRA001324/CRR042722 } \\
\text { NGDC, Genome Sequence Archive } \\
\text { https://bigd.big.ac.cn/gsa/browse/CRA001324/CRR042723 }\end{array}$ \\
\hline Data file 2 [29-31] & $\begin{array}{l}\text { b_L3_1_clean.fq.gz } \\
\text { b_L3_2_clean.fq.gz } \\
\text { b_L4_1_clean.fq.gz } \\
\text { b_L4_2_clean.fq.gz } \\
\text { b_L6_1_clean.fq.gz } \\
\text { b_L6_2_clean.fq.gz }\end{array}$ & FASTQ (fq.gz) & $\begin{array}{l}\text { NGDC, Genome Sequence Archive } \\
\text { https://bigd.big.ac.cn/gsa/browse/CRA001324/CRR042724 } \\
\text { NGDC, Genome Sequence Archive } \\
\text { https://bigd.big.ac.cn/gsa/browse/CRA001324/CRR042725 } \\
\text { NGDC, Genome Sequence Archive } \\
\text { https://bigd.big.ac.cn/gsa/browse/CRA001324/CRR042726 }\end{array}$ \\
\hline Data file 3 [32-34] & $\begin{array}{l}\text { 8-a_L5_1_clean.fq.gz } \\
\text { 8-a_L5_2_clean.fq.gz } \\
\text { 8-a_L6_1_clean.fq.gz } \\
\text { 8-a_L6_2_clean.fq.gz } \\
\text { 8-a_L8_1_clean.fq.gz } \\
\text { 8-a_L8_2_clean.fq.gz }\end{array}$ & FASTQ (fq.gz) & $\begin{array}{l}\text { NGDC, Genome Sequence Archive } \\
\text { https://bigd.big.ac.cn/gsa/browse/CRA001324/CRR042727 } \\
\text { NGDC, Genome Sequence Archive } \\
\text { https://bigd.big.ac.cn/gsa/browse/CRA001324/CRR042728 } \\
\text { NGDC, Genome Sequence Archive } \\
\text { https://bigd.big.ac.cn/gsa/browse/CRA001324/CRR042729 }\end{array}$ \\
\hline Data file 4 [35-37] & 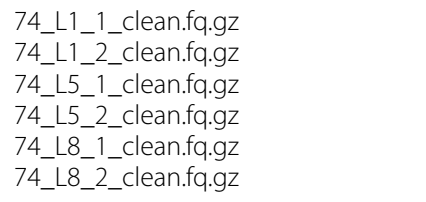 & FASTQ (fq.gz) & $\begin{array}{l}\text { NGDC, Genome Sequence Archive } \\
\text { https://bigd.big.ac.cn/gsa/browse/CRA001324/CRR042730 } \\
\text { NGDC, Genome Sequence Archive } \\
\text { https://bigd.big.ac.cn/gsa/browse/CRA001324/CRR042731 } \\
\text { NGDC, Genome Sequence Archive } \\
\text { https://bigd.big.ac.cn/gsa/browse/CRA001324/CRR042732 }\end{array}$ \\
\hline Data file 5 [38-41] & $\begin{array}{l}\text { 85_L5_1_clean.fq.gz } \\
\text { 85_L5_2_clean.fq.gz } \\
\text { 85_L6_1_clean.fq.gz } \\
\text { 85_L6_2_clean.fq.gz } \\
\text { 85_L7_1_clean.fq.gz } \\
\text { 85_L7_2_clean.fq.gz } \\
\text { 85_L8_1_clean.fq.gz } \\
\text { 85_L8_2_clean.fq.gz }\end{array}$ & FASTQ (fq.gz) & $\begin{array}{l}\text { NGDC, Genome Sequence Archive } \\
\text { https://bigd.big.ac.cn/gsa/browse/CRA001324/CRR042733 } \\
\text { NGDC, Genome Sequence Archive } \\
\text { https://bigd.big.ac.cn/gsa/browse/CRA001324/CRR042734 } \\
\text { NGDC, Genome Sequence Archive } \\
\text { https://bigd.big.ac.cn/gsa/browse/CRA001324/CRR042735 } \\
\text { NGDC, Genome Sequence Archive } \\
\text { https://bigd.big.ac.cn/gsa/browse/CRA001324/CRR042736 }\end{array}$ \\
\hline $\begin{array}{l}\text { Data file } 6 \\
\text { [42-45] }\end{array}$ & $\begin{array}{l}\text { 1_L1_1_clean.fq.gz } \\
\text { 1_L1_2_clean.fq.gz } \\
\text { 1_L2_1_clean.fq.gz } \\
\text { 1_L2_2_clean.fq.gz } \\
\text { 1_L3_1__clean.fq.gz } \\
\text { 1_L3_2_clean.fq.gz } \\
\text { 1_L4_1_clean.fq.gz } \\
\text { 1_L4_2_clean.fq.gz }\end{array}$ & FASTQ (fq.gz) & $\begin{array}{l}\text { NGDC, Genome Sequence Archive } \\
\text { https://bigd.big.ac.cn/gsa/browse/CRA001324/CRR042737 } \\
\text { NGDC, Genome Sequence Archive } \\
\text { https://bigd.big.ac.cn/gsa/browse/CRA001324/CRR042738 } \\
\text { NGDC, Genome Sequence Archive } \\
\text { https://bigd.big.ac.cn/gsa/browse/CRA001324/CRR042739 } \\
\text { NGDC, Genome Sequence Archive } \\
\text { https://bigd.big.ac.cn/gsa/browse/CRA001324/CRR042740 }\end{array}$ \\
\hline
\end{tabular}


$1,884,054,828$ short reads were generated for all of the six individuals. After filtering, the range of total highquality sequence data was from $42.1 \mathrm{~Gb}$ to $51 \mathrm{~Gb}$ and the coverage varied from $14.51 \mathrm{X}$ to $17.15 \mathrm{X}$. The range of the mean insert sizes and their standard deviations in the sequenced data for all samples was from 280.06 to 331.86 and from 27.12 to 33.94 , respectively.

The quality assessment of raw sequence reads was done with FastQC (http://www.bioinformatics.babraham. ac.uk/projects/fastqc/). We used BWA (v.0.7.15) [15] program to compare sequence data with the reference genome (CanFam3.1) downloaded from the Ensembl (http://asia.ensembl.org/Canis_lupus_familiaris/Info/ Index). The alignment quality was assessed with SAMtools v.1.9 using flagstat and depth commands [16]. The short-read sequences with the mean depth of $16 \mathrm{X}$ were mapped to the dog reference genome (CanFam3.1) and achieved 99\% coverage of the reference assembly. The mapping output files were preprocessed using SAMtools [16], the Picard tools (http://broadinstitute.githu b.io/picard/) and GATK tools [17]. We used variome detection pipeline for this data using CNVnator [18], BreakDancer [19], DELLY [20] and Bedtools [21] programs [14]. Finally, we compared the effect of variome between the dog and wolf genomes using Sorting Intolerant from Tolerant (SIFT) algorithm [19], Ensembl annotation [22] and DAVID [23] tool [14]. The data presented herein together with our previously mitochondrial DNA sequence on Iranian dogs [11] will provide useful resources to understand genetic structure of the Iranian dogs and testing hypotheses on the dog origin and domestication issues.

\section{Limitations}

Sample size for the dog and wolf populations is a limitation of our work. We could create genome sequence data from only three wolves and three dogs. In addition, we produced the short-reads with a mean depth of $16 \mathrm{X}$ which is a medium depth and it might not be suitable for some genomic analyses.

\section{Abbreviations}

FC: Fertile crescent; GSA: Genome sequence archive; NCBI: National Center for Biotechnology Information; NGDC: National Genomics Data Center; SIFT: Sorting intolerant from tolerant.

\section{Acknowledgements}

We are thankful for helping personnel from Department of Environmental Protection in Iran, office of natural resources in Kerman and Tehran, Shiraz, Tehran Eram and Kerman zoological gardens and the dog owners. Also, we greatly appreciate Dr. Iman Memarian and Dr. Hosein Rashidi for sampling from wolf in Tehran Eram and Kerman zoological gardens.

\section{Authors' contributions}

$A E$ and $Y-P Z$ designed the experiment. Sampling was done by ZAG and MAF. ZAG carried out DNA extraction. The genome resequencing data were created and assessed by GDW and ZAG. AE, GDW and MAF read the manuscript. All authors read and approved the final manuscript.

\section{Funding}

The funds for conducting this experiment were provided by the Youth Innovation Promotion Association, Chinese Academy of Sciences, the Chinese Academy of Sciences President's International Fellowship Initiative (No. 2016VBA050), the National Natural Science Foundation of China (No. 91531303), the International Cooperation Program of Bureau of International Cooperation of Chinese Academy of Sciences (No.GJHZ1559) and the Animal Branch of the Germplasm Bank of Wild Species, Chinese Academy of Sciences (the Large Research Infrastructure Funding).

\section{Availability of data and materials}

The raw data reported here are available in the NGDC, GSA database (https ://bigd.big.ac.cn/gsa/) under the accession number of CRA001324 and NCBI under the accession of PRJNA639312. Please see the data files 1 to 6 in Table 1 for more details on the raw sequence data [24-45].

\section{Ethics approval and consent to participate}

This work had Institutional Animal Care and Use Committee (Kunming Institute of Zoology, approval ID: SYDW-2013021) approval. We collected peripheral blood samples from 3 Iranian dogs with the consent of owners and 3 gray wolves after obtaining consent for research from the Department of Environmental Protection in Iran (No. 93/34089, dated 14 October 2014).

\section{Consent for publication}

Not applicable.

\section{Competing interests}

The authors declare that they have no competing interests.

\section{Author details}

${ }^{1}$ Department of Animal Science, Faculty of Agriculture, Shahid Bahonar University of Kerman, PB 76169-133 Kerman, Iran. ${ }^{2}$ Young Researchers Society, Shahid Bahonar University of Kerman, PB 76169-133 Kerman, Iran. ${ }^{3}$ State Key Laboratory of Genetic Resources and Evolution, Kunming Institute of Zoology, Chinese Academy of Sciences, No. 32 Jiaochang Donglu, Kunming 650223, Yunnan, China. ${ }^{4}$ State Key Laboratory for Conservation and Utilization of Bio-Resources in Yunnan, Yunnan University, Kunming 650091, China.

Received: 2 April 2020 Accepted: 4 September 2020

Published online: 16 September 2020

\section{References}

1. Clutton-Brock J. Domesticated animals: from early times, Heinemann in assoc. with British Museum. London: Natural history; 1981.

2. Wang GD, Zhai W, Yang HC, Wang L, Zhong L, Liu YH, et al. Out of southern East Asia: the natural history of domestic dogs across the world. Cell Res. 2016:26:21-33.

3. Clutton-Brock J. Origins of the dog: domestication and early history. In: Serpell J, editor. The domestic dog: its evolution, behaviour, and interactions with people. New York: Cambridge University Press; 1995. p. 7-20.

4. Vilà C, Savolainen P, Maldonado JE, Amorim IR, Rice JE, Honeycutt $\mathrm{RL}$, et al. Multiple and ancient origins of the domestic dog. Science. 1997;276:1687-9

5. Wayne RK. Molecular evolution of the dog family. Trends Genet. 1993;9:218-24

6. Colledge S, Conolly J, Shennan S, Bellwood P, Bouby L, Hansen J, et al. Archaeobotanical evidence for the spread of farming in the Eastern Mediterranean 1. Curr Anthropol. 2004;45:S35-58.

7. Zeder MA. Domestication and early agriculture in the Mediterranean Basin: origins, diffusion, and impact. Proc Natl Acad Sci U S A. 2008;105:11597-604.

8. Alizadeh $\mathrm{A}$. The rise of the highland Elamite state in southwestern Iran. Curr Anthropol. 2010;51:353-83. 
9. Przezdziecki XJB, Paris G. Our levriers: the past, present and future of all sighthounds. France: Les Amis de Xavier Przezdziecki, La Colle-sur-Loup; 2001.

10. VonHoldt BM, et al. Genome-wide SNP and haplotype analyses reveal a rich history underlying dog domestication. Nature. 2010;464:898-902.

11. Amiri Ghanatsaman Z, Adeola AC, Asadi Fozi M, Ma YP, Peng MS, Wang GD, et al. Mitochondrial DNA sequence variation in Iranian native dogs. Mitochondrial DNA A DNA Mapp Seq Anal. 2017;17:1-9.

12. Ardalan $A$, Kluetsch $C F$, Zhang AB, Erdogan $M$, Uhlén $M$, Houshmand $M$, et al. Comprehensive study of mtDNA among Southwest Asian dogs contradicts independent domestication of wolf, but implies dog-wolf hybridization. Ecol Evol. 2011;1:373-85.

13. Freedman AH, Gronau I, Schweizer RM, Ortega-Del Vecchyo D, Han E, Silva PM, et al. Genome sequencing highlights the dynamic early history of dogs. PLoS Genet. 2014;10:e1004016.

14. Amiri Ghanatsaman Z, Wang GD, Asadollahpour Nanaei H, Asadi Fozi M, Peng MS, Esmailizadeh A, et al. Whole genome resequencing of the Iranian native dogs and wolves to unravel variome during dog domestication. BMC Genomics. 2020;21:1-11.

15. Li H, Durbin R. Fast and accurate short read alignment with burrowswheeler transform. Bioinformatics. 2009;25:1754-60.

16. Li H, Handsaker B, Wysoker A, Fennell T, Ruan J, Homer N, et al. The sequence alignment/map format and SAMtools. Bioinformatics. 2009;25:2078-9.

17. McKenna A, Hanna M, Banks E, Sivachenko A, Cibulskis K, Kernytsky A, et al. The genome analysis toolkit: a MapReduce frame work for analyzing nextgeneration DNA sequencing data. Genome Res. 2010;20:1297-303.

18. Abyzov A, Urban AE, Snyder M, Gerstein M. CNVnator: an approach to discover, genotype, and characterize typical and atypical CNVs from family and population genome sequencing. Genome Res. 2011;21:974-84.

19. Chen K, Wallis J, McLellan M, et al. BreakDancer: an algorithm for highresolution mapping of genomic structural variation. Nat Methods. 2009:6:677-81.

20. Rausch T, Zichner T, Schlattl A, Stütz AM, Benes V, Korbel JO. DELLY: structural variant discovery by integrated paired-end and split-read analysis. Bioinformatics. 2012;28:i333-9.

21. Quinlan AR, Hall IM. BEDTools: a flexible suite of utilities for comparing genomic features. Bioinformatics. 2010;26:841-2.

22. Flicek P, Amode MR, Barrell D, Beal K, Brent S, Carvalho-Silva D, et al. Ensembl 2012. Nucleic Acids Res. 2011;40:D84-90.

23. Huang DW, Sherman BT, Lempicki RA. Systematic and integrative analysis of large gene lists using DAVID bioinformatics resources. Nat Protoc. 2009;4:44-57.

24. BIGD Genome Warehouse; 2020. https://bigd.big.ac.cn/bioproject/brows e/PRJCA001183.

25. BIGD Genome Warehouse; 2020. https://bigd.big.ac.cn/gsa/browse/ CRA001324/CRR042720.
26. BIGD Genome Warehouse; 2020. https://bigd.big.ac.cn/gsa/browse/ CRA001324/CRR042721.

27. BIGD Genome Warehouse; 2020. https://bigd.big.ac.cn/gsa/browse/ CRA001324/CRR042722.

28. BIGD Genome Warehouse; 2020. https://bigd.big.ac.cn/gsa/browse/ CRA001324/CRR042723.

29. BIGD Genome Warehouse; 2020. https://bigd.big.ac.cn/gsa/browse/ CRA001324/CRR042724.

30. BIGD Genome Warehouse; 2020. https://bigd.big.ac.cn/gsa/browse/ CRA001324/CRR042725.

31. BIGD Genome Warehouse; 2020. https://bigd.big.ac.cn/gsa/browse/ CRA001324/CRR042726.

32. BIGD Genome Warehouse; 2020. https://bigd.big.ac.cn/gsa/browse/ CRA001324/CRR042727.

33. BIGD Genome Warehouse; 2020. https://bigd.big.ac.cn/gsa/browse/ CRA001324/CRR042728.

34. BIGD Genome Warehouse; 2020. https://bigd.big.ac.cn/gsa/browse/ CRA001324/CRR042729.

35. BIGD Genome Warehouse; 2020. https://bigd.big.ac.cn/gsa/browse/ CRA001324/CRR042730.

36. BIGD Genome Warehouse; 2020. https://bigd.big.ac.cn/gsa/browse/ CRA001324/CRR042731.

37. BIGD Genome Warehouse; 2020. https://bigd.big.ac.cn/gsa/browse/ CRA001324/CRR042732.

38. BIGD Genome Warehouse; 2020. https://bigd.big.ac.cn/gsa/browse/ CRA001324/CRR042733.

39. BIGD Genome Warehouse; 2020. https://bigd.big.ac.cn/gsa/browse/ CRA001324/CRR042734.

40. BIGD Genome Warehouse; 2020. https://bigd.big.ac.cn/gsa/browse/ CRA001324/CRR042735.

41. BIGD Genome Warehouse; 2020. https://bigd.big.ac.cn/gsa/browse/ CRA001324/CRR042736.

42. BIGD Genome Warehouse; 2020. https://bigd.big.ac.cn/gsa/browse/ CRA001324/CRR042737.

43. BIGD Genome Warehouse; 2020. https://bigd.big.ac.cn/gsa/browse/ CRA001324/CRR042738.

44. BIGD Genome Warehouse; 2020. https://bigd.big.ac.cn/gsa/browse/ CRA001324/CRR042739.

45. BIGD Genome Warehouse; 2020. https://bigd.big.ac.cn/gsa/browse/ CRA001324/CRR042740.

\section{Publisher's Note}

Springer Nature remains neutral with regard to jurisdictional claims in published maps and institutional affiliations.
Ready to submit your research? Choose BMC and benefit from:

- fast, convenient online submission

- thorough peer review by experienced researchers in your field

- rapid publication on acceptance

- support for research data, including large and complex data types

- gold Open Access which fosters wider collaboration and increased citations

- maximum visibility for your research: over 100M website views per year

At BMC, research is always in progress.

Learn more biomedcentral.com/submissions 